\title{
VIRGIN OLIVE OIL PHENOLS-SPECTROSCOPIC EVALUATION IN BASIC MEDIUM: ANALYSIS OF TOTAL CONTENT OF HYDROXYTYROSOL
}

\author{
MUSTAFA CITTAN ${ }^{\mathrm{a}^{*}}$
}

\begin{abstract}
Here a spectrophotometric technique in the visible region combined with an environmental friendly ultra-pure water based liquid-liquid extraction procedure has been described for the determination of total hydroxytyrosol in virgin olive oils regardless of whether it is free or combined. Determination of total hydroxytyrosol was carried out where the absorption peak of the colored compounds formed with autopolymerization of quinones that occured following the oxidation of hydroxytyrosol and its secoiridoid derivatives in a basic medium was measured as an analytical signal. The method was linear in a concentration range of $0.2-15.0 \mathrm{mg} \mathrm{L}^{-1}$ with a correlation coefficient of 0.9990 . Detection and quantification limits were 0.05 and $0.18 \mathrm{mg} \mathrm{L}^{-1}$, respectively. Intra-day and inter-day precision studies indicated that the proposed method was repeatable. In addition, the liquidliquid extraction procedure was quite efficient with recovery values between 108 and $117 \%$. Finally, the proposed method was successfully applied to the analysis of total hydroxytyrosol in a virgin olive oil and the results were compared with those obtained by a method existing in the literature for the determination of total hydroxytyrosol based on acid hydrolysis of secoiridoid aglycons followed by liquid chromatography-tandem mass spectrometry.
\end{abstract}

Keywords: hydroxytyrosol; virgin olive oil; phenolic compounds; visible spectroscopy; liquid-liquid extraction.

\section{INTRODUCTION}

It is well established that the Mediterranean diet (MD) is very effective against: cancer [1], cardiovascular disease [2], inflammation [3], diabetes [4], and aging [5]. Many of the benefits associated with the MD are the result of

\footnotetext{
a Manisa Celal Bayar University, Faculty of Science and Letters, Department of Chemistry, 45140, Manisa, Turkey

*Corresponding author: mustafa.cittan@cbu.edu.tr
} 
a high intake of antioxidants and anti-inflammatory elements present in several components of this diet [6]. Virgin olive oil (VOO) is an essential component of the MD and is directly obtained from ripe olive fruits using only mechanical extraction. The beneficial effects of $\mathrm{VOO}$ can be attributed to the high relationship between unsaturated and saturated fatty acids and also to the antioxidant properties of its phenolic composition [7].

The VOO phenolic fraction is mainly comprised by phenyl ethyl alcohols (hydroxytyrosol, tyrosol) and their secoiridoids derivates $[8,9]$, linked to the aldehydic and dialdehydic forms of elenolic acid (such as oleuropein, the dialdehydic form of decarboxymethyl elenolic acid linked to hydroxytyrosol (3,4-DHPEA-EDA), 4-(acetoxyethyl)-1,2-dihydroxybenzene (3,4-DHPEA-AC), dialdehydic form of the decarboxymethyl elenolic acid linked to tyrosol ( $p$-HPEAEDA), p-4-hydroxyphenylethanol-elenolic acid, an isomer of ligstroside aglycone (p-HPEA-EA), 3,4-dihydroxyphenylethanol-elenolic acid, an isomer of oleuropein aglycone (3,4-DHPEA-EA) and its methylated form (methyl 3,4-DHPEAEA)) [10]. Some other minor components are also commonly present, like: phenolic acids (such as ferulic, p-coumaric, vanillic, caffeic, hydroxyphenylacetic, gentisic, gallic, homovanillic and cinnamic acid) $[8,11]$, lignans (pinoresinol and 1-acetoxypinoresinol) [12], flavonoids (luteolin and apigenin) [13], and aldehydes (vanillin) [14].

The origin of hydroxytyrosol $(\mathrm{HT})$ is the hydrolysis of oleuropein which happens during the ripening of the olives, and during the storage and elaboration of table olives [15]. Among olive oil phenolic compounds, HT has been the most investigated compound, primarily for its bioavailability [16] and is the most potent as antioxidant [17] and the oxygen radical absorbance capacity (ORAC) of HT is twice that observed for oleuropein, three and ten times more than for epicatechin and ascorbic acid, respectively [18]. HT also leads to apoptosis and growth arrest in vitro [19], including melanoma cells [20], HL60 leukaemia cells [21], and colon cancer cell lines [22].

Numerous techniques have been applied for the extraction of phenolic compounds from VOO. A simple and fast methanol-n-hexane-based liquidliquid extraction (LLE) was usually carried out to extract the polar fraction of VOO, especially the phenolic compounds [23]. On the other hand, solid phase extraction (SPE) [24,25], ultrasound-assisted extraction (UAE) [26], and liquidliquid micro extraction techniques (LLME) $[10,26]$ have all been followed for this purpose. Further, the extraction of phenolic compounds from VOO by deep eutectic solvents (DESs) has been recently reported [27].

The determination of HT in VOO was usually carried out using chromatographic techniques. High performance liquid chromatography (HPLC) technique by ultraviolet (UV) detection [28], fluorescence (FL) detection [29], 
and mass spectrometric (MS) detection [30,31] were followed for this purpose. Gas chromatography-mass spectroscopy (GC-MS) [32] and capillary zone electrophoresis (CZE) equipped with diode array detector (DAD) [33] techniques were also used to determine HT in VOO.

Most of the beneficial properties of VOO have been attributed to HT and, to a lesser extent, to tyrosol (Tyr). However, the analysis of the total content of HT in VOOs fails to provide reliable data because of the unreliable quantification of secoiridoid derivatives. As there are no commercial standards available regarding these secoiridoid aglycons, it is difficult to define the derivatives and determine the accurate total amount of HT in VOO. On the other hand, a method has been described to determine the total content of HT and Tyr via acid hydrolysis process of aglycons with $2 \mathrm{M} \mathrm{HCl}$ in extraction step followed by HPLC-DAD analysis [12].

In this work, a spectrophotometric technique in the visible region combined with a simple environmental friendly ultra-pure water based LLE is proposed for the determination of total HT as HT equivalent in VOOs regardless of whether it is free or combined. The approach to determining total $\mathrm{HT}$ in this paper exploits the oxidation of diphenols in a basic medium $(0.1 \mathrm{M} \mathrm{NaOH}$ solution) to the corresponding quinones and then absorbance measurement of the colored compounds formed by autopolymerization of the quinones [34]. This is the first report that provides the mass spectrum of the resulting colored compounds that proves the autopolymerization of diphenols in basic medium. A diagrammed illustration of the complete method was depicted in Scheme 1. The paper also provides a detailed investigation of the interference effects of the other potential VOO phenolics to the methodology by using the individual standards of 34 phenolic compounds. Consequently, the paper is quite original since it suggests a simple analytical technique combined with an environmental friendly ultra-pure water based liquid-liquid extraction procedure to determine the total contents of HT in VOOs without the interference of other predominant phenolic compounds, Tyr and its derivatives (monophenol derivatives in VOOs). The proposed method was applied for the determination of total HT in a VOO and the obtained result was compared with those obtained via the extraction process of acid hydrolysis of the aglycons proposed in the literature followed by liquid chromatography-electrospray tandem mass spectrometry (LC-ESI-MS/MS) technique [12]. The method would be a simple, cheap and rapid alternative to determine the total HT in VOOs. 


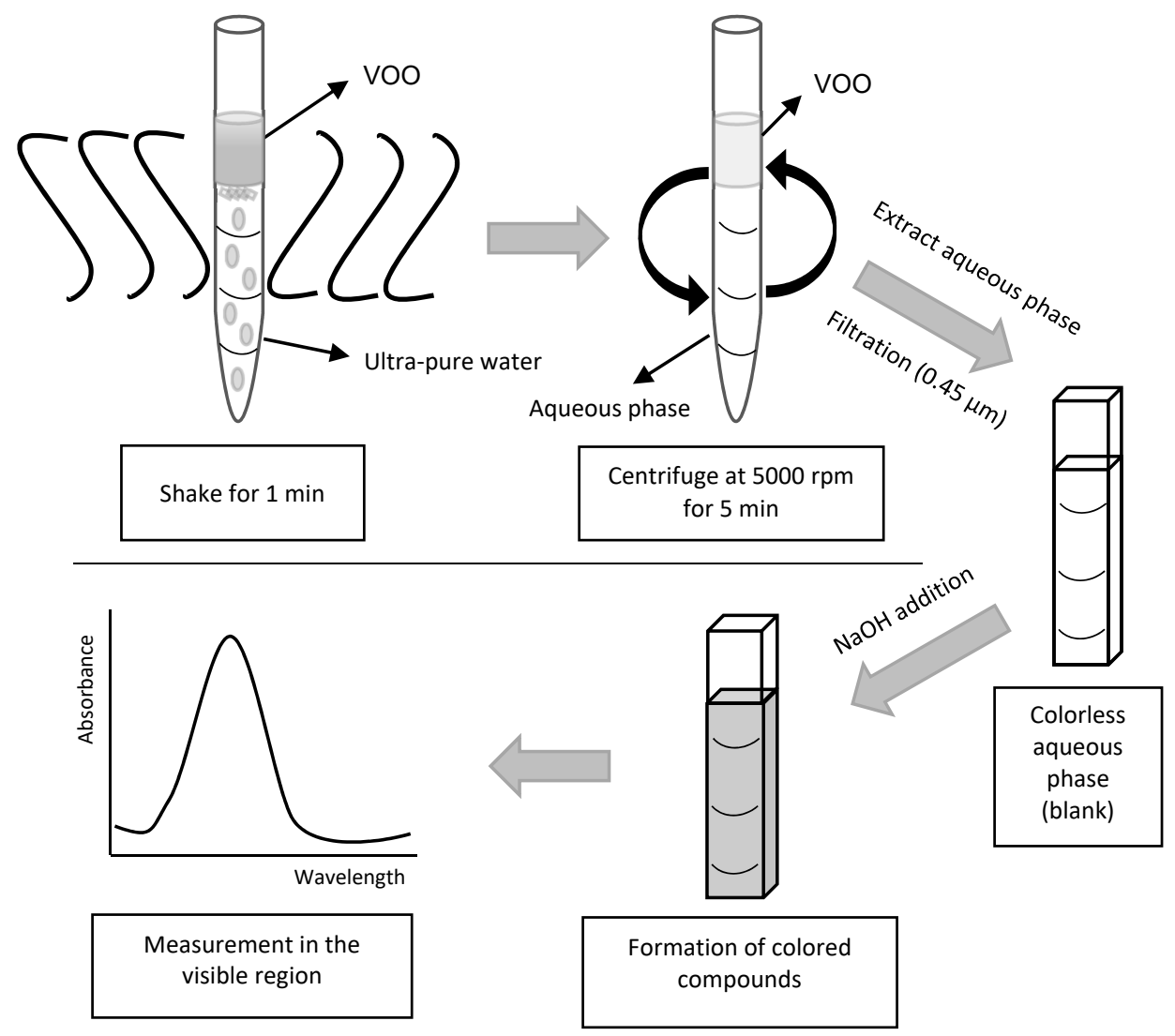

Scheme 1. The diagrammed illustration of total hydroxytyrosol analysis in virgin olive oil.

\section{RESULTS AND DISCUSSION}

\section{Optimization of $\mathrm{NaOH}$ concentration}

The $\mathrm{NaOH}$ concentration of the medium was increased from 0.01 to $1 \mathrm{M}$ in order to obtain high absorption intensity. Figure 1 shows the effect of the $\mathrm{NaOH}$ concentration on the response of $5 \mathrm{mg} \mathrm{L}^{-1} \mathrm{HT}$ at $530 \mathrm{~nm}$. The determination was performed quadruplicate for each concentration point. The results clearly demonstrated that the sensitivity increased up to $0.1 \mathrm{M} \mathrm{NaOH}$ concentration. On the other hand, higher $\mathrm{NaOH}$ concentrations not only reduced the sensitivity of the method, but also caused higher relative standard deviations in the analyte response. Therefore, the medium was $0.1 \mathrm{M} \mathrm{NaOH}$. 


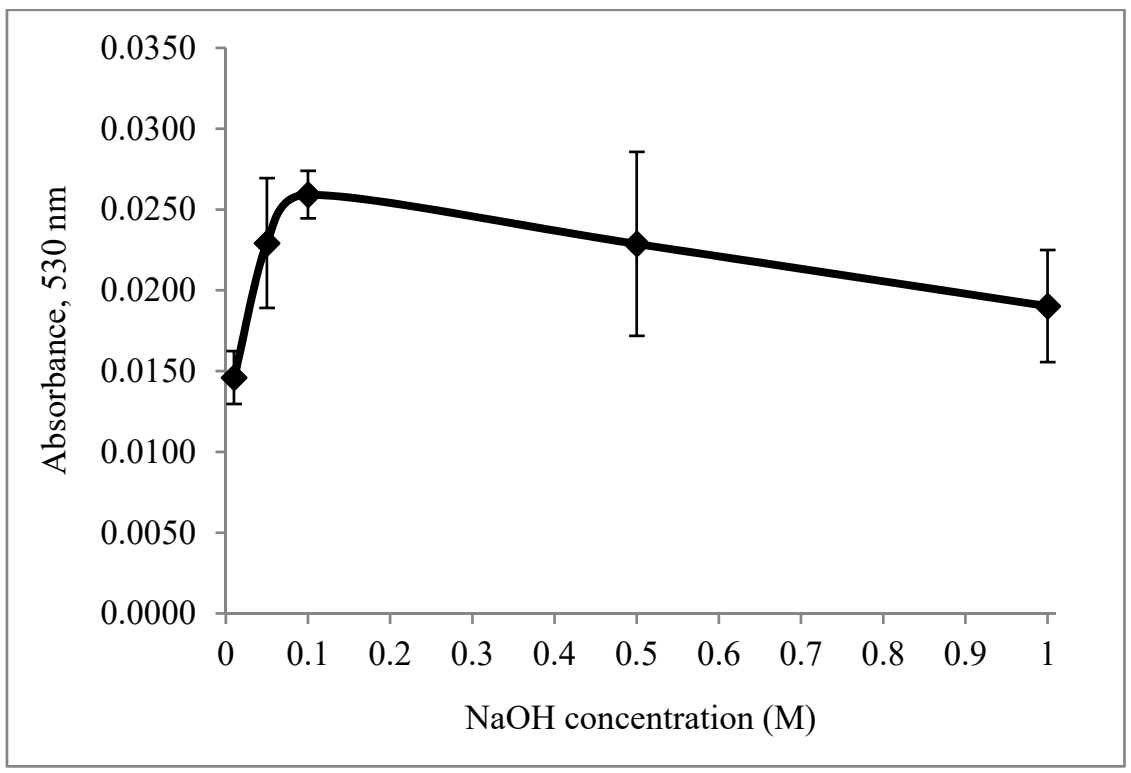

Figure 1. Effect of $\mathrm{NaOH}$ concentration on the analytical signal of hydroxytyrosol.

\section{Selectivity of the proposed analysis method}

34 different phenolic compounds (homovanillic acid, trans-cinnamic acid, taxifolin, (+)-catechin, pyrocatechol, (-)-epicatechin, ferulic acid, chlorogenic acid, caffeic acid, gentisic acid, 3-hydroxybenzoic acid, 4-hydroxybenzoic acid, p-coumaric acid, sinapic acid, gallic acid, syringic acid, vanillin, rosmarinic acid, protocatechuic acid, 2-hydroxycinnamic acid, 3,4-dihydroxyphenylacetic acid, vanillic acid, apigenin, luteolin, apigenin 7-glucoside, luteolin 7-glucoside, eriodictyol, pinoresinol, tyrosol, verbascoside, kaempferol, hyperoside, hesperidin and quercetin) were used to evaluate the selectivity of the proposed analytical method for the determination of total $\mathrm{HT}$. To this end, the behavior of the phenolic compounds in a concentration of $50 \mathrm{mg} \mathrm{L}^{-1}$ in $0.1 \mathrm{M} \mathrm{NaOH}$ medium was observed in 350 to $800 \mathrm{~nm}$ wavelength range using the individual commercial analytical standards of the compounds one by one.

The approach to the proposed analysis method exploits the oxidation of diphenols in a basic medium $(0.1 \mathrm{M} \mathrm{NaOH}$ solution) to the corresponding quinones and absorbance measurement of the colored compounds formed by autopolymerization of the quinones. Since the method was specific only to the compounds containing more than one hydroxyl group in the phenolic rings, monophenols such as Tyr, 3-hydroxybenzoic acid, 4-hydroxybenzoic acid, 
sinapic acid, vanillic acid, ferulic acid, syringic acid, vanillin, 2-hydroxycinnamic acid, p-coumaric acid, pinoresinol, hesperidin, trans-cinnamic acid and homovanillic acid, as expected, did not react with $\mathrm{NaOH}$ to form colored compounds. Thus, Tyr and its secoiridoid derivatives, the other major components of the VOO, did not interfere with the determination of HT by the proposed method. In addition, quercetin, kaempferol, chlorogenic acid, hyperoside, luteolin, apigenin, luteolin 7-glucoside, and apigenin 7-glucoside exhibited considerable absorbance at a wavelength range of $380-430 \mathrm{~nm}$ in $0.1 \mathrm{M} \mathrm{NaOH}$ medium. Nevertheless, they did not significantly interfere with the determination of HT, as the absorbance of HT was recorded at $530 \mathrm{~nm}$ wavelength.

Protocatechuic acid, gentisic acid, 3,4-dihydroxyphenylacetic acid, gallic acid, (+)-catechin, (-)-epicatechin, pyrocatechol, caffeic acid, rosmarinic acid, eriodictyol, taxifolin, and verbascoside absorbed similar wavelengths with HT in $0.1 \mathrm{M} \mathrm{NaOH}$ medium which resulted significant interferences to the methodology. The phenolic content of $\mathrm{VOO}$ has been described numerous times in the literature and, no study has been reported that VOO contains (+)catechin, (-)-epicatechin, pyrocatechol, verbascoside, rosmarinic acid and eriodictyol. On the other hand, gallic acid [11,35], protocatechuic acid [35], 3,4-dihydroxyphenylacetic acid [29,35], gentisic acid [11,29,35], caffeic acid $[26,30,36]$ and taxifolin [35] have been reported in VOO. However, the reported results prove that these compounds are present at only trace levels in VOO compared to hydroxytyrosol, tyrosol and their secoiridoid derivatives $[10,29,30]$. As a result, when the matrix is $\mathrm{VOO}$, it is considered that the proposed method is quite reliable for total HT determination without the interference of the other VOO phenolics, especially Tyr and its secoiridoid derivatives.

\section{Linearity, limit of detection and quantification}

The linearity was examined by establishing the external standard calibration curves in a concentration range of $0.2-15.0 \mathrm{mg} \mathrm{L}^{-1}$. All calibration curves were generated from the stock standard solution of HT with three replicates per level at 7 different concentrations $(0.2,0.5,1.0,3.0,5.0,10.0$ and $15.0 \mathrm{mg} \mathrm{L}^{-1}$ ) in $0.1 \mathrm{M} \mathrm{NaOH}$ solution. Well defined absorption peaks were observed (maximum absorbance at approximately $495 \mathrm{~nm}$ wavelength) in Figure 2A. But the absorbance of HT was recorded at $530 \mathrm{~nm}$ throughout the study to prevent possible interference of the phenolic compounds that exhibited considerable absorbance at a wavelength range of $380-430 \mathrm{~nm}$ in $0.1 \mathrm{M} \mathrm{NaOH}$ medium (see section selectivity of the proposed analysis method). The plot of the calibration curve (Figure 2B) was found to be linear with a 0.9990 correlation coefficient. 
VIRGIN OLIVE OIL PHENOLS-SPECTROSCOPIC EVALUATION IN BASIC MEDIUM:

ANALYSIS OF TOTAL CONTENT OF HYDROXYTYROSOL
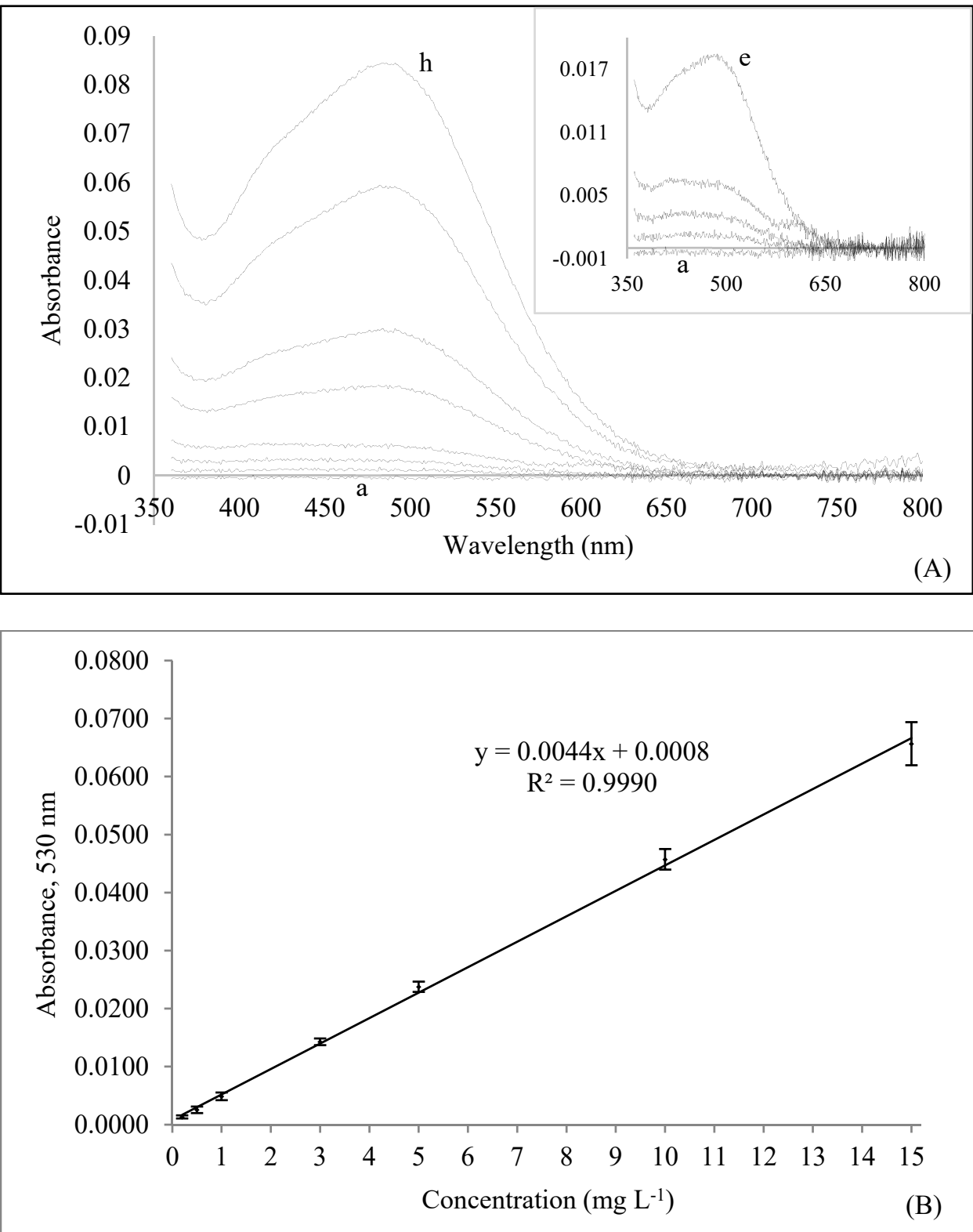

Figure 2. (A) Visible absorption spectrums of various concentrations of hydroxytyrosol; (a-h): blank, 0.2, 0.5, 1.0, 3.0, 5.0, 10.0 and $15.0 \mathrm{mg} \mathrm{L}^{-1}$. Inset showed visible absorption spectrums of low concentrations; (a-e): blank, $0.2,0.5,1.0$ and $3.0 \mathrm{mg} \mathrm{L}^{-1}$. (B) The calibration curve for hydroxytyrosol. 
The limit of detection (LOD) and limit of quantification (LOQ) were calculated using the formula $L O D=3 S D / b$ and $L O Q=10 S D / b$, respectively, where SD is the standard deviation of ten reagent blank determinations and $b$ is the slope of the calibration curve. The LOD and LOQ were 0.05 and $0.18 \mathrm{mg} \mathrm{L}^{-1}$, respectively.

\section{Repeatability and precision}

Calibration standards at three different concentrations of 1.0, 3.0 and $10.0 \mathrm{mg} \mathrm{L}^{-1}$ were used to determine the intra-day (three repetitions of each concentration) and inter-day (three repetitions of each concentration and three days) repeatability of proposed method. The results are shown in Table 1. As expected, the intra-day precision was higher than the inter-day precision in all cases and the method showed a good overall repeatability (the intraday relative standard deviations (RSDs) were lower than $2.1 \%$ and lower than $13.5 \%$ for inter-day assays) when the HT concentration was above $1.0 \mathrm{mg} \mathrm{L}^{-1}$.

Table 1. Intra-day and inter-day precision of the method

\begin{tabular}{|c|c|c|}
\hline \multirow{2}{*}{$\begin{array}{c}\text { Concentration } \\
\left(\mathrm{mg} \mathrm{L}^{-1}\right)\end{array}$} & \multicolumn{2}{|c|}{ RSD (\%) } \\
\cline { 2 - 3 } & Intra-day $(\mathrm{n}=3)$ & Inter-day $(\mathrm{n}=9)$ \\
\hline 1.0 & 2.1 & 13.5 \\
\hline 3.0 & 1.6 & 4.0 \\
\hline 10.0 & 0.8 & 3.9 \\
\hline
\end{tabular}

RSD, relative standard deviation.

\section{Recovery of the proposed liquid-liquid extraction procedure}

UAE technique using methanol-water mixture as extractant is one of the most effective aid to extract the phenolic compounds from foodstuffs [3739]. To this end, proposed LLE technique using only ultra-pure water only and UAE method were followed comparatively to determine the efficiency of the proposed extraction technique for the extraction of HT from VOO. Both extracts obtained with LLE and UAE techniques were scanned for the phenolic contents by a modified LC-ESI-MS/MS method described previously [40] (include: HT and the other phenolics used in the selectivity studies except Tyr, homovanillic acid and trans-cinnamic acid, due to their incompatibility with the mobile phase system used; totally 33 individual phenolic compounds) and only $\mathrm{HT}, p$-coumaric acid, pinoresinol, luteolin and apigenin were detected in the extracts. The chromatograms are given in Figure 3 . The results clearly showed that the proposed LLE method was more effective for the extraction of $\mathrm{HT}$ and $p$-coumaric acid from VOO. On the other hand, the LLE method 
failed to extract the pinoresinol, luteolin and apigenin when compared to the UAE method. When considering luteolin and apigenin exhibited considerable absorbance at approximately $400-410 \mathrm{~nm}$ wavelength in the spectrophotometric measurements, it was suggested that the LLE method using only ultra-pure water as extractant is more compatible with the proposed analysis method.

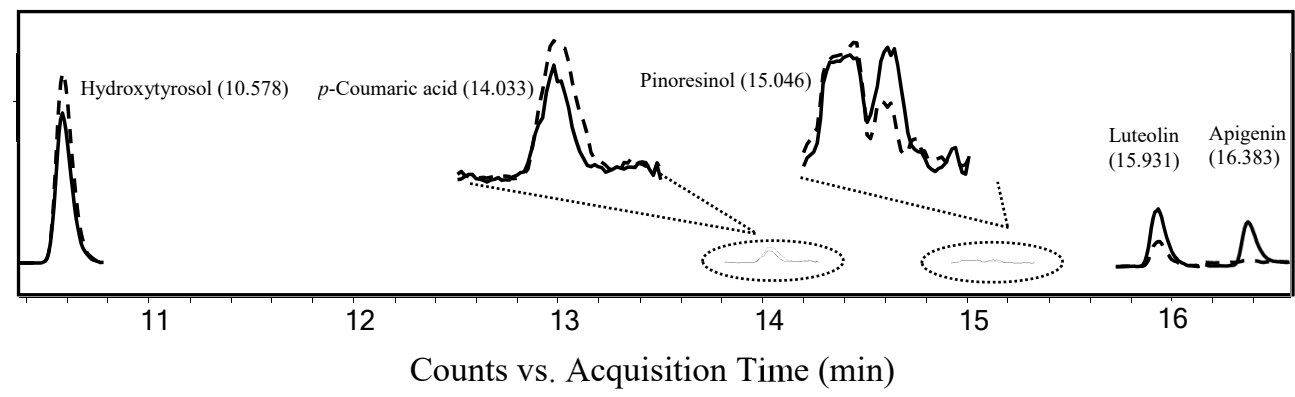

Figure 3. LC-ESI-MS/MS MRM chromatograms of the virgin olive oil extracts (ultrasound-assisted extraction using methanol/water mixture as extractant: $\ldots$, ultra-pure water based liquid-liquid extraction: _ _ _ _ -).

Furthermore, recovery of the proposed LLE technique was evaluated by spiking $1 \mathrm{~mL}$ of VOO sample at 10.0 and $100.0 \mathrm{mg} \mathrm{L}^{-1}$ concentration levels of HT standard (three repetitions of each concentration). The spiked samples were extracted via LLE procedure and analyzed $(n=3)$ with LC-ESI-MS/MS. Quantitative recoveries were obtained in all cases, varying from 108 to $117 \%$ (Table 2). As a result, the proposed LLE method is not only efficient for HT extraction, but also environmental friendly because of using only ultra-pure water in the extraction step.

Table 2. Recovery of the proposed liquid-liquid extraction method for hydroxytyrosol

\begin{tabular}{|c|c|c|c|c|}
\hline \multirow{2}{*}{$\begin{array}{c}\text { Content } \\
\left(\mathrm{mg} \mathrm{L}^{-1}\right)\end{array}$} & $\begin{array}{c}\text { Spiked } \\
\left(\mathrm{mg} \mathrm{L}^{-1}\right)\end{array}$ & $\begin{array}{c}\text { Found } \\
\left(\mathrm{mg} \mathrm{L}^{-1}\right)\end{array}$ & $\begin{array}{c}\text { Recovery } \\
(\%)\end{array}$ & $\begin{array}{c}\text { Mean } \\
(\%)\end{array}$ \\
\hline \multirow{3}{*}{$16.6 \pm 0.5$} & \multirow{3}{*}{10.0} & 28.4 & 118 & \\
\cline { 3 - 4 } & & 26.7 & 101 & \multirow{2}{*}{$108 \pm 9$} \\
\cline { 3 - 4 } & \multirow{2}{*}{100.0} & 27.2 & 106 & \\
\cline { 3 - 4 } & & 138.0 & 121 & \multirow{2}{*}{$117 \pm 4$} \\
\cline { 3 - 4 } & & 131.4 & 115 & \\
\cline { 3 - 4 } & & 130.1 & 114 & \\
\hline
\end{tabular}




\section{Quantitative analysis of virgin olive oil sample}

$1 \mathrm{~mL}$ of VOO samples were extracted simultaneously via LLE and acid hydrolysis procedures to determine the total $\mathrm{HT}$ content of the sample by the proposed spectrophotometric and LC-ESI-MS/MS method, respectively. The extracts were analyzed in triplicate using the relevant analysis method to compare the accuracy of the proposed spectrophotometric technique. The aqueous extracts were used as blank in spectrophotometric measurements. Then, appropriate volume of $3 \mathrm{M} \mathrm{NaOH}$ was added to each aqueous extract for a final concentration of $0.1 \mathrm{M}$ before measuring absorbance at a wavelength of $530 \mathrm{~nm}$.

A typical calibration curve obtained using a series of standard solutions over the concentration range from 25.0 to $500.0 \mathrm{\mu g} \mathrm{L}^{-1}$ used for total HT determination in VOO sample by the LC-ESI-MS/MS method is shown in Figure 4. On the other hand, Figure 5 shows the standard addition (2.0, 4.0 and $6.0 \mathrm{mg} \mathrm{L}^{-1} \mathrm{HT}$ ) spectrums and calibration curve obtained for the VOO sample. The results were in agreement, $18.0 \pm 0.1 \mathrm{mg} \mathrm{L}^{-1}$ for the proposed spectrophotometric technique combined with a simple ultra-pure water based LLE and $17.5 \pm 0.5 \mathrm{mg} \mathrm{L}^{-1}$ for the LC-ESI-MS/MS method following the acid hydrolysis process.

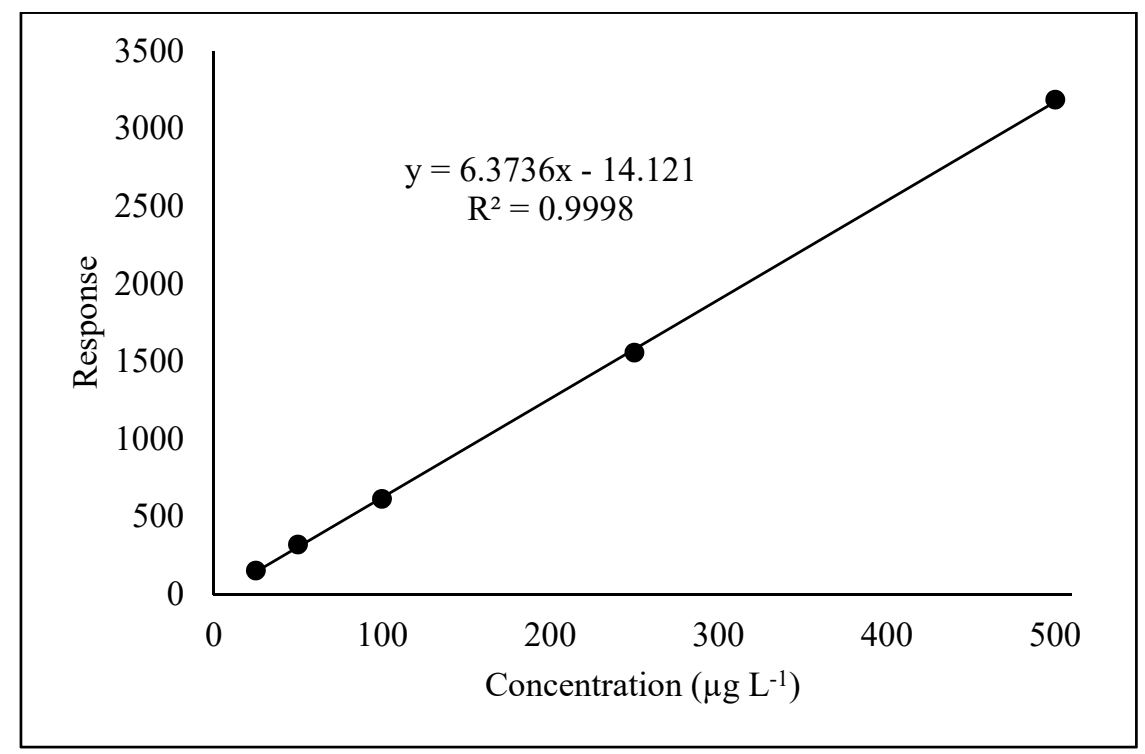

Figure 4. Typical calibration curve of hydroxytyrosol in LC-ESI-MS/MS method. 
VIRGIN OLIVE OIL PHENOLS-SPECTROSCOPIC EVALUATION IN BASIC MEDIUM: ANALYSIS OF TOTAL CONTENT OF HYDROXYTYROSOL

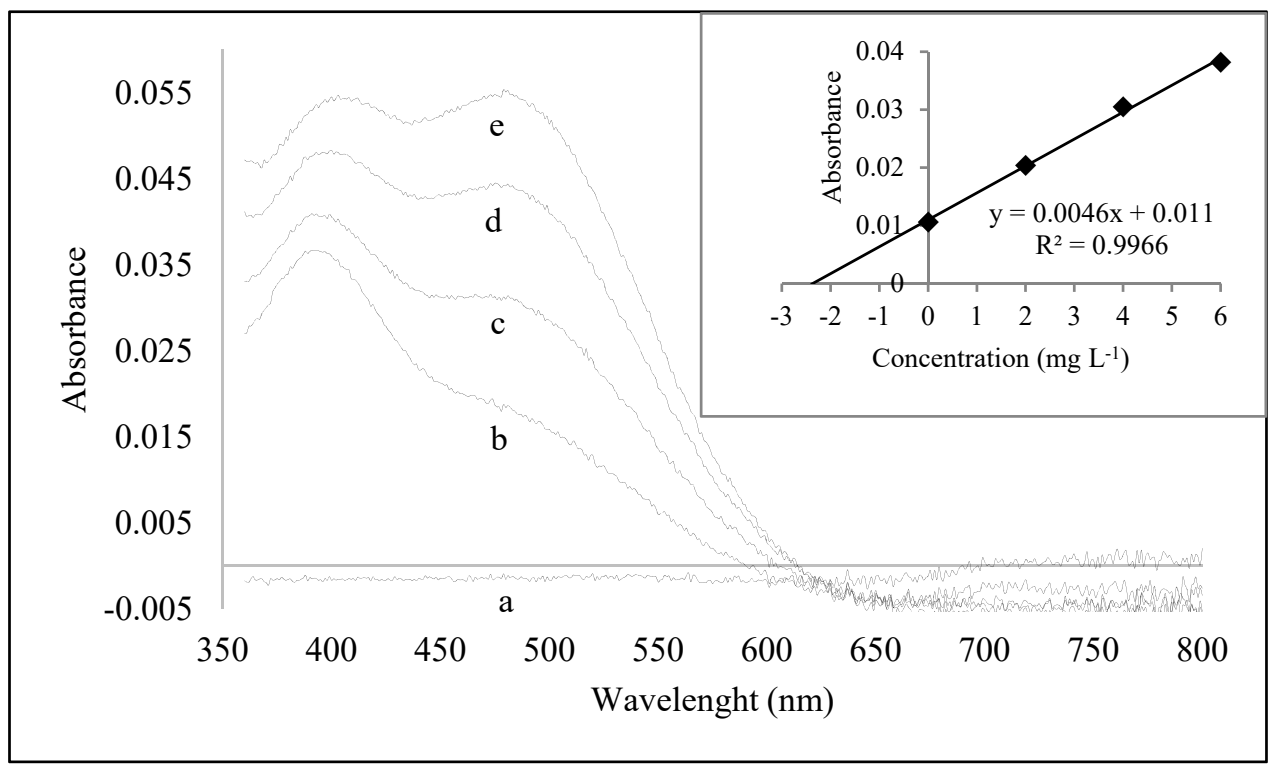

Figure 5. Visible absorption spectrums of (a) blank, (b) sample, after addition of (c) 2, (d) 4 and (e) $6 \mathrm{mg} \mathrm{L}^{-1}$ hydroxytyrosol standard. The standard addition calibration curve is inset.

\section{Matrix effect}

Matrix effect was evaluated to ensure the bias-free analytical results. Due to absence of blank matrices for VOOs, it was determined at three different concentrations $\left(2.0,4.0\right.$ and $\left.10.0 \mathrm{mg} \mathrm{L}^{-1}\right)$ of HT. The matrix effect was calculated relatively by comparing the absorbance measurement of three spectrums [41]. The Eq. (1) is given as:

$$
\mathrm{M} E(\%)=\left[\frac{\mathrm{A}(\text { matrix }+\mathrm{std})-\mathrm{A}(\text { matrix })}{\mathrm{A}(\mathrm{std})}-1\right] \times 100(\%)
$$

where $A($ matrix + std $)$ is the response for the matrix with the addition of standard; $A$ (matrix) is the response for the matrix only; and $A(s t d)$ is the response for the standard only. 
The mean matrix effect was $1.4 \%$ with a standard deviation of $1.0 \%$, which suggested that a very slight signal enhancement observed. On the other hand, the matrix effect was also assessed by comparing the slopes of standard calibration curve and standard addition method. Good agreement between the slopes of both curves (Figure 2B and 5) and the calculated matrix effect were proved that there was no significant matrix effect when the sample preparation was carried out by the proposed LLE method.

\section{Mass spectrometric evaluation of the resulting colored solution}

The mass spectrum (Figure 6 ) of the colored solution formed by the proposed method was evaluated in order to clarify the autopolymerization of the quinones that occurs with the oxidation of diphenols in a basic medium by direct infusion of $\mathrm{HT}$ of $50 \mathrm{mg} \mathrm{L}^{-1}$ concentration in $0.1 \mathrm{M} \mathrm{NaOH}$ in to the mass spectrometer in the 50 to 2200 amu mass range. The ions at $\mathrm{m} / \mathrm{z} 757.7$, 929.0, and $1082.1 \mathrm{amu}$, defined as integer multiples of the HT molecular weight $\left(154.16 \mathrm{~g} \mathrm{~mol}^{-1}\right)$ in the mass spectrum, support the described autopolymerization approach.

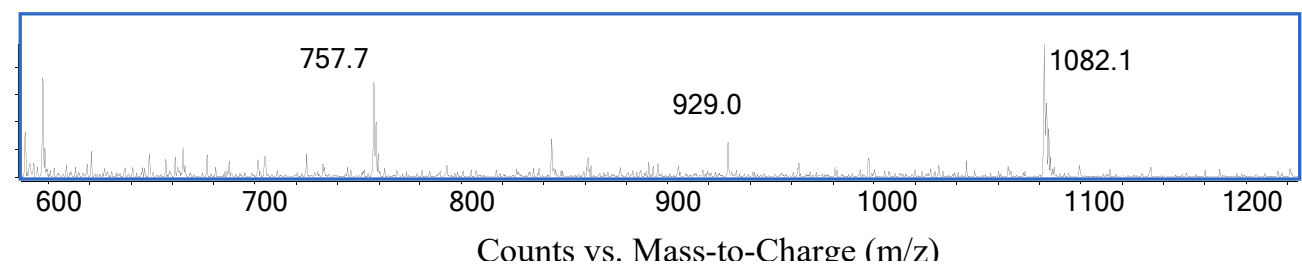

Figure 6. Mass spectrum of the resulting colored solution.

\section{CONCLUSIONS}

It is more useful to choose simple and cheap analytical techniques in matrices where the analyte is the main component. In such cases, trace compounds do not interfere significantly with the determination of the major analyte. Thus, the amount of analyte in the matrix can be easily estimated by using simple and cheap analytical techniques as an alternative to existing complicated methods.

HT and Tyr are the main phenolic compounds in VOO. Many beneficial effects of VOO have been attributed to HT and, to a lesser extent, to Tyr. The proposed method in the present study proved to be simple, low- 
cost, effective, and suitable for determining total HT in VOOs without the interference of Tyr and its derivatives (the other predominant components of VOOs). The method is also quite important since it determines the total HT as $\mathrm{HT}$ equivalent regardless of whether it is free or combined.

The proposed technique was linear in a concentration range of $0.2-$ $15.0 \mathrm{mg} \mathrm{L}^{-1}$ with a correlation coefficient of 0.9990 . The limit of detection and quantification of the method were obtained as 0.05 and $0.18 \mathrm{mg} \mathrm{L}^{-1}$, respectively. The method showed a good overall repeatability (the intra-day RSDs were lower than $2.1 \%$ and lower than $13.5 \%$ for inter-day assays) when the HT concentration was above $1.0 \mathrm{mg} \mathrm{L}^{-1}$. Environmental friendly ultra-pure based LLE of HT and its derivatives had good recovery values between 108 and $117 \%$. No significant matrix effect was observed. Finally, a detailed investigation of the interference effects of the other potential VOO phenolics to the methodology was carried out and it was suggested that the proposed analytical technique is quite reliable and easily applicable for total $\mathrm{HT}$ determination when the test matrix is VOO.

\section{EXPERIMENTAL}

\section{Apparatus}

Absorption spectra were recorded in an ultraviolet and visible (UV-Vis) spectrophotometer (Agilent Technologies, Cary 60). An Agilent Technologies 1260 Infinity liquid chromatography system hyphenated to a 6420 Triple Quad mass spectrometer was used for LC analyses. The ultrasound cleaning bath was Daihan, WUC-D10H. A WiseShake SHO-2D digital orbital shaker was used for the agitation in acid hydrolysis process.

\section{Reagents}

All commercial phenolic standards were purchased from SigmaAldrich (St. Louis, MO, USA), Fluka (St. Louis, MO, USA) and HWI Analytik (Ruelzheim, Germany). In addition, hydrochloric acid and methanol were purchased from Sigma-Aldrich (St. Louis, MO, USA). Formic acid and sodium hydroxide were obtained from Merck (Darmstadt, Germany). Ultrapure water $(18 \mathrm{~m} \Omega$ ) was obtained from a Milli-Q water purification system (Millipore Co., Ltd.). Stock solutions of the phenolic compounds were prepared in methanol. 


\section{LC-ESI-MS/MS method}

Phenolic determination of VOOs was carried out following the procedure described in our previous study by using a Poroshell 120 EC-C18 $(100 \mathrm{~mm}$ x $4.6 \mathrm{~mm}$ I.D., $2.7 \mu \mathrm{m}$ ) column [40].

\section{Assays for the extraction of phenolic compounds}

Extraction of phenolic compounds from VOOs were carried out using (i) a simple, environmental friendly ultra-pure water based LLE procedure that combined with the proposed spectrophotometric method, (ii) an UAE procedure that mentioned as an effective technique in the literature using methanol/water (80:20, v/v) mixture as extractant and, (iii) an acid hydrolysis process of aglycons to determine the total contents of HT described elsewhere [12]. Figure 7 shows the scheme for the three processes of the extraction methods.

Liquid-liquid extraction

(LLE)
Ultrasound-assisted extraction (UAE)

Acid hydrolysis process

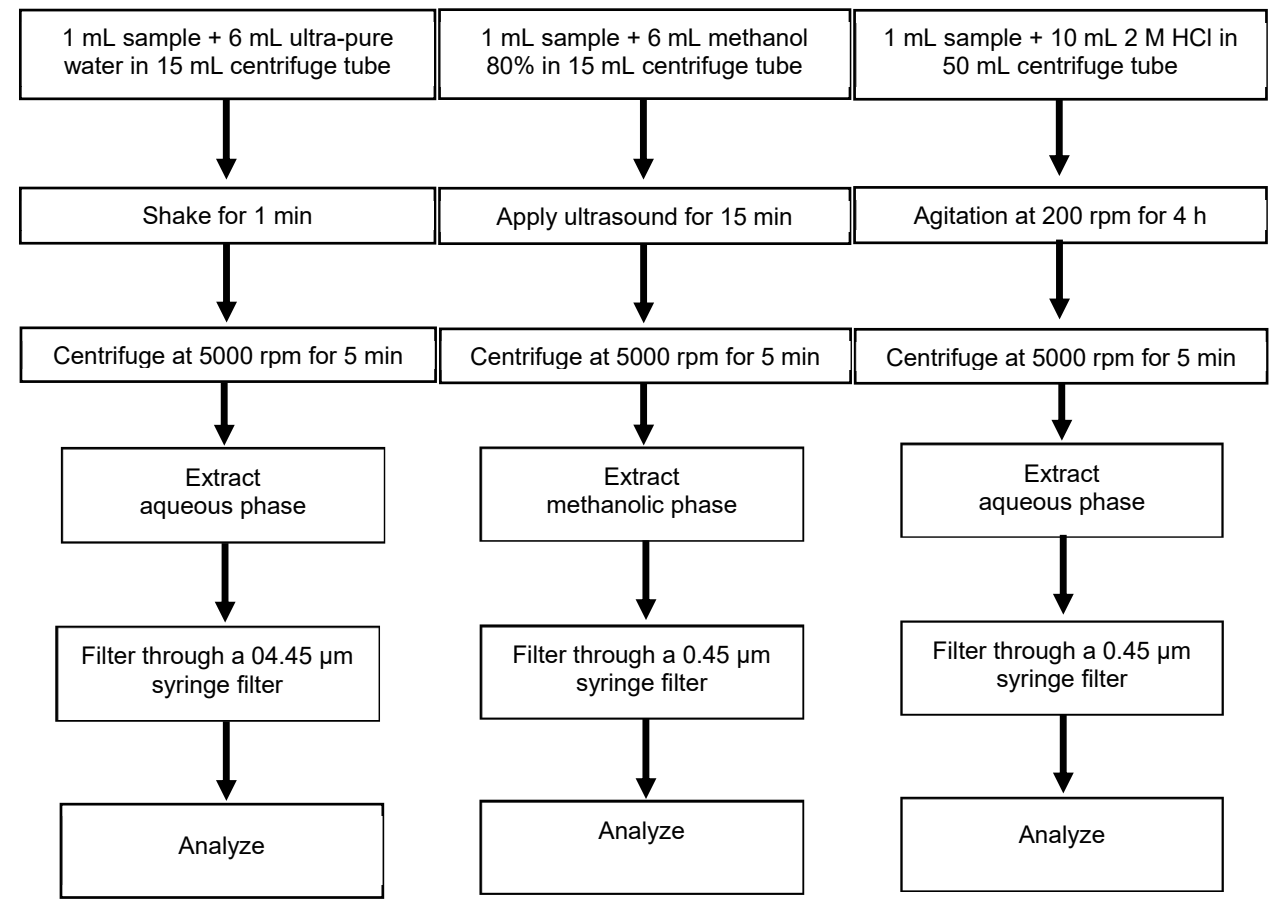

Figure 7. Scheme of the extraction methods followed in the study. 
VIRGIN OLIVE OIL PHENOLS-SPECTROSCOPIC EVALUATION IN BASIC MEDIUM: ANALYSIS OF TOTAL CONTENT OF HYDROXYTYROSOL

Ultra-pure water based liquid-liquid extraction procedure

VOO $(1 \mathrm{~mL})$ samples were extracted with $6 \mathrm{~mL}$ of ultra-pure water in a polyethylene centrifuge tube $(15 \mathrm{~mL}$, conical bottom). The centrifuge tubes were sealed tightly and shaken vigorously for $1 \mathrm{~min}$ by hand. The content of the tube was then centrifuged at $3000 \mathrm{rpm}$ for $5 \mathrm{~min}$. Finally, the aqueous phase was filtered through a $0.45 \mu \mathrm{m}$ syringe filter prior to analysis.

\section{Ultrasound-assisted extraction procedure}

VOO $(1 \mathrm{~mL})$ was placed into polyethylene centrifuge tube $(15 \mathrm{~mL}$, conical bottom) containing $6 \mathrm{~mL}$ of methanol/water mixture $(80: 20, \mathrm{v} / \mathrm{v})$ and directly sonicated using an ultrasonic bath for $15 \mathrm{~min}$. The content of the tube was then centrifuged at $3000 \mathrm{rpm}$ for $5 \mathrm{~min}$ and the extracts were filtered through a $0.45 \mu \mathrm{m}$ syringe filter prior to analysis.

\section{Acid hydrolysis process}

$1 \mathrm{~mL}$ of $\mathrm{VOO}$ was weighed in in a polyethylene centrifuge tube (50 $\mathrm{mL}$, conical bottom) and $10 \mathrm{~mL}$ of $2 \mathrm{M} \mathrm{HCl}$ was added. The mixture was vigorously homogenized by agitation at $200 \mathrm{rpm}$ in the orbital shaker for 4 hours. After the centrifugation process at $3000 \mathrm{rpm}$ for $5 \mathrm{~min}$, the aqueous phase was filtered through a $0.22 \mu \mathrm{m}$ pore size.

\section{Method validation}

A validation procedure was performed in order to establish the analytical performance features of the proposed method, ensuring the successful quantification of total HT in VOOs, evaluating linearity, selectivity, limit of detection and quantification, precision (intra and inter-day), matrix effect and extraction recovery.

\section{REFERENCES}

[1] E. Couto, P. Boffetta, P. Lagiou, P. Ferrari, G. Buckland, K. Overvad, C. C. Dahm, A. Tjønneland, A. Olsen, F. Clavel-Chapelon et al., Br. J. Cancer. 2011, 104 (9), 1493-1499.

[2] R. Estruch, M. A. Martínez-González, D. Corella, J. Salas-Salvadó, V. RuizGutiérrez, M. I. Covas, M. Fiol, E. Gómez-Gracia, M. C. López-Sabater, E. Vinyoles et al., Ann. Intern. Med. 2006, 145 (1), 1-11.

[3] M. A. Martínez-González, J. Salas-Salvadó, R. Estruch, D. Corella, M. Fitó, E. Ros, Prog. Cardiovasc. Dis. 2015, 58 (1), 50-60. 
[4] J. Salas-Salvadó, M. Bulló, R. Estruch, E. Ros, M. I. Covas, N. Ibarrola-Jurado, D. Corella, F. Arós, E. Gómez-Gracia, V. Ruiz-Gutiérrez et al., Ann. Intern. Med. 2014, 160 (1), 1-10.

[5] A. Varela-Lopez, P. Bullon, M. Battino, Mc. Ramirez-Tortosa, J. J. Ochoa, M. D. Cordero, C. L. Ramirez-Tortosa, C. Rubini, A. Zizzi, J. L. Quiles, Journals Gerontol. - Ser. A Biol. Sci. Med. Sci. 2016, 71 (5), 593-600.

[6] M. Robles-Almazan, M. Pulido-Moran, J. Moreno-Fernandez, C. Ramirez-Tortosa, C. Rodriguez-Garcia, J. L. Quiles, Mc. Ramirez-Tortosa, Food Res. Int. 2018, 105, 654-667.

[7] E. N. Frankel, J. Agric. Food Chem. 2010, 58 (10), 5991-6006.

[8] K. L. Tuck, P. J. Hayball, J. Nutr. Biochem. 2002, 13 (11), 636-644.

[9] V. Lavelli, L. Bondesan, J. Agric. Food Chem. 2005, 53 (4), 1102-1107.

[10] M. Becerra-Herrera, M. Sánchez-Astudillo, R. Beltrán, A. Sayago, LWT - Food Sci. Technol. 2014, 57 (1), 49-57.

[11] M. I. Alarcón Flores, R. Romero-González, A. Garrido Frenich, J. L. Martínez Vidal, Food Chem. 2012, 134 (4), 2465-2472.

[12] C. Romero, M. Brenes, J. Agric. Food Chem. 2012, 60 (36), 9017-9022.

[13] P. Reboredo-Rodríguez, L. Rey-Salgueiro, J. Regueiro, C. González-Barreiro, B. Cancho-Grande, J. Simal-Gándara, Food Chem. 2014, 150, 128-136.

[14] M. J. Tovar, M. J. Motilva, M. P. Romero, J. Agric. Food Chem. 2001, 49 (11), $5502-5508$.

[15] S. Charoenprasert, A. Mitchell, J. Agric. Food Chem. 2012, 60 (29), 7081-7095.

[16] A. Incani, M. Deiana, G. Corona, K. Vafeiadou, D. Vauzour, M. A. Dessì, J. P. E. Spencer, Mol. Nutr. Food Res. 2009, 54 (6), 788-796.

[17] R. Owen, A. Giacosa, W. Hull, R. Haubner, B. Spiegelhalder, H. Bartsch, Eur. J. Cancer. 2000, 36 (10), 1235-1247.

[18] A. Pastor, J. Rodríguez-Morató, E. Olesti, M. Pujadas, C. Pérez-Mañá, O. Khymenets, M. Fitó, M.-I. Covas, R. Solá, M.-J. Motilva et al., J. Chromatogr. A. 2016, 1437, 183-190.

[19] E. Terzuoli, A. Giachetti, M. Ziche, S. Donnini, Mol. Nutr. Food Res. 2016, 60 (3), 519-529.

[20] S. D'Angelo, D. Ingrosso, V. Migliardi, A. Sorrentino, G. Donnarumma, A. Baroni, L. Masella, M. Antonietta Tufano, M. Zappia, P. Galletti, Free Radic. Biol. Med. 2005, 38 (7), 908-919.

[21] R. Fabiani, A. De Bartolomeo, P. Rosignoli, M. Servili, G. F. Montedoro, G. Morozzi, Eur. J. Cancer Prev. 2002, 11 (4), 351-358.

[22] G. Corona, M. Deiana, A. Incani, D. Vauzour, M. A. Dessì, J. P. E. Spencer, Mol. Nutr. Food Res. 2009, 53 (7), 897-903.

[23] F. M. Pirisi, P. Cabras, C. F. Cao, M. Migliorini, M. Muggelli, J. Agric. Food Chem. 2000, 48 (4), 1191-1196.

[24] R. Mateos, J. L. Espartero, M. Trujillo, J. J. Ríos, M. León-Camacho, F. Alcudia, A. Cert, J. Agric. Food Chem. 2001, 49 (5), 2185-2192.

[25] M. N. Franco, T. Galeano-Díaz, Ó. López, J. G. Fernández-Bolaños, J. Sánchez, C. De Miguel, M. V. Gil, D. Martín-Vertedor, Food Chem. 2014, 163, 289-298. 
VIRGIN OLIVE OIL PHENOLS-SPECTROSCOPIC EVALUATION IN BASIC MEDIUM: ANALYSIS OF TOTAL CONTENT OF HYDROXYTYROSOL

[26] M. L. Pizarro, M. Becerra, A. Sayago, M. Beltrán, R. Beltrán, Food Anal. Methods. 2013, 6 (1), 123-132.

[27] A. García, E. Rodríguez-Juan, G. Rodríguez-Gutiérrez, J. J. Rios, J. FernándezBolaños, Food Chem. 2016, 197, 554-561.

[28] M. Tasioula-Margari, O. Okogeri, Food Chem. 2001, 74 (3), 377-383.

[29] M. P. Godoy-Caballero, M. I. Acedo-Valenzuela, T. Galeano-Díaz, Talanta. 2012, $101,479-487$.

[30] M. Suárez, A. Macià, M.-P. Romero, M.-J. Motilva, J. Chromatogr. A. 2008, 1214 (1-2), 90-99.

[31] M. I. Alarcón Flores, R. Romero-González, A. Garrido Frenich, J. L. Martínez Vidal, Food Chem. 2012, 134 (4), 2465-2472.

[32] M. Tasioula-margari, O. Okogeri, J. Food Sci. 2001, 66 (4), 530-534.

[33] M. Bonoli, M. Montanucci, T. Gallina Toschi, G. Lercker, J. Chromatogr. A. 2003, $1011(1-2), 163-172$.

[34] H. Hotta, M. Ueda, S. Nagano, Y. Tsujino, J. Koyama, T. Osakai, Anal. Biochem. 2002, 303 (1), 66-72.

[35] A. Carrasco Pancorbo, C. Cruces-Blanco, A. Segura Carretero, A. Fernández Gutiérrez, J. Agric. Food Chem. 2004, 52 (22), 6687-6693.

[36] S. Kesen, H. Kelebek, S. Selli, J. Am. Oil Chem. Soc. 2014, 91 (3), 385-394.

[37] F. Priego-Capote, J. Ruiz-Jiménez, M. Luque de Castro, J. Chromatogr. A. 2004, 1045 (1-2), 239-246.

[38] Y.-Q. Ma, J.-C. Chen, D.-H. Liu, X.-Q. Ye, Ultrason. Sonochem. 2009, 16 (1), $57-62$.

[39] E. Espada-Bellido, M. Ferreiro-González, C. Carrera, M. Palma, C. G. Barroso, G. F. Barbero, Food Chem. 2017, 219, 23-32.

[40] M. Cittan, A. Çelik, J. Chromatogr. Sci. 2018, 56 (4), 336-343.

[41] R. Wu, F. Ma, L. Zhang, P. Li, G. Li, Q. Zhang, W. Zhang, X. Wang, Food Chem. 2016, 204, 334-342. 